\title{
QUALITY ASSURANCE IN TEACHER EDUCATION
}

\author{
Harmandeep Singh , Arjinder Kaur \\ Assistant Professor P.G.Deptt. of C.sc \& I.T, Khadur Sahib-143117 \\ Assistant Professor SGAD College of Eduaction Khadur Sahib-143117
}

ABSTRACT: Quality' became very common word nowadays. What actually quality means. It is very familiar term but its meanings and uses vary widely. In general term quality is the position of a 'product' or a 'process' attribute on good bad scale. It is often associated or linked with defects and deficiencies in products or process.

Keywords: Education; Teacher; Quality

\section{INTRODUCTION:}

"Quality is the capability of products or services to knowingly satisfy those preconceived composite wants of the user(s) that are intelligently related to the characteristics of performance, and do not cause major overt or covert reactions or actions by other people." Johnson -1987

"Fitness of use." Juran -1974

"Conformance to requirements." Crosby - 1984

From the above concepts of quality when two aspects combined together may help in understanding the concept in an optimum way.

- Relates to the features and attributes of the product or service.

- Absence of deficiencies in the products.

Thus quality can be defined as the totality of features and characteristics of a product or service that bear on its ability to satisfy stated or implied needs. Alexander Astin (1993) pointed out in the discussion on quality in education system,

You - cannot - define - it - but - you - know - it - when - you - see - it.

According to him there are four views on excellence in quality higher education-

- Excellence in reputation

- Excellence in resources

- Excellence in content

- Excellence in outcome

Educational institutions have a tendency to measure their quality in terms of students' academic achievement. The assumption is that if the results of an institution are improving, its quality must be good. Besides, academic achievement is not a competent measure of educational quality since it depends on many other factors such as students innate intelligence, study habits, learning styles, motivation, parental socio-economic status and home environment. Hence we have to identify relevant indicators of educational quality and measure it in a reliable and valid manner.

In quality management system total quality management starts with- 


\section{Quality inspection}

This is very initial step and through this, defects are observed and communicated for quality improvement.

Quality improvement

It is the commitment and approach used to continuously improve every process in every part of an organization. Quality improvement is long term approach, established through incremental steps. Quality improvement is a way of working that facilitates change and influences teaching and learning by quality providing the stimulus and support for further assurance of quality.

\section{Quality Assurance}

Once quality is assured at reasonable level it needs to be controlled from deviations to ensure quality management. In the TQM the goods or services are expected to be with zero defects and when require further modification. Due to social or professional reasons the same may be communicated at the appropriate level for compliance. Thus quality assurance acts a backbone in the total system of quality management and hence requires more concern.

The subject of quality assurance has become a important word in the sphere of higher education. Among higher education teacher education occupies central stage due to its being the caretaker of macro educational system. The teacher education has assign the task of producing the teachers for primary and secondary education and also build solid foundation of the system.Due to its role and significance, the teacher education has vast amount of growth during last decade. Education of teachers is not only responsible for improvement of school education but also for preparing competent, committed and professionally well qualified teachers who can meet the demands of a system. A teacher's content knowledge, verbal skills or enthusiasm for learning necessarily constitutes high mark for being a high quality teacher. Viewed in this perspective, a high quality teacher must imbibe the four pillars of education as mentioned in the Jacques Delor's report, "Learning the Treasure Within" (1996). These four pillars of education are,

Learning to know

The teachers must have an urge for learning to learn. It can be achieved by combining a sufficiently broad general knowledge with the opportunity to work in depth on a small number of subjects.

*Learning to do

Emphasizes acquisition not only of an occupational skill but also of the competence to deal with many situations and work in teams.

*Learning to live together

Requires development of an understanding of other people and an appreciation of interdependence in a sprit of respect for the values of pluralism, mutual understanding and peace.

*Learning to be

Will support the development of one's personality and ability to act with greater autonomy, judgement and personal responsibility. Thus these four pillars are consider as a founding pillars in the preparation of high quality teacher.

Also high quality teacher must posses three C's,

\section{${ }^{*}$ Competence}


It is concerned with identifying the basic competencies in the teacher which will have the potential and promise for ensuring results and delivering the goods.

\section{${ }^{*}$ Commitment}

It will imply lot of self-governance and self-initiative of the teacher.

\section{${ }^{*}$ Confidence}

The teacher perceives instrumentality and their utilization for improving the day-to-day situation.

However with the expansion of teacher education institutes the assurance of quality has been challenged which is the basic requirement in knowledge society. Due emergence of knowledge society the need of quality assurance has further multiplied its intensity. The application of quality assurance in industrial sector helps us designing quality assurance strategies for teacher education. i) Maintain ii) Enhancing iii) Ownership iv) Checking quality As a whole quality assurance in teacher education may be ensured by studying its customer than assuring quality at every stage because all these parts are interrelated. i) The education service (Value added learning) The value added learning in teacher education is complex of inputs to the teaching and learning, teaching process, service quality, and institutional arrangement to assure quality in the inputs the discipline may use national curricula with inbuilt flexibility suiting to regional requirements. The disseminator of teacher education is a teacher and preparing the teachers where he/she is in the direct contact with the end users. The close interaction between the service provider and end user also allow multiple opportunities for feedback and evaluation directing at satisfaction level of the end users. However due to involvement of repeated human interactions the possibility of human errors also enhances. Therefore, for the purpose of assuring quality in teacher education we need to infuse soft indicators of quality assurance i.e. care. Courtesy, concern, friendliness, and helpfulness to delivery superior service quality.

ii) Primary external customer (learners) They are future teachers i.e. nation builders hence entry norm should be made more reliable. Once student has been enrolled in the teacher education programme again it is the teachers who can make him feel the real worth of the course. The trainee teachers should be prepared socially responsible teachers with high values and morality. After completion of degree students should enjoy feeling of self- sufficacy and fulfillment. iii) Secondary external customers(parents, society) Parents are preliminary school for children and the prime decision maker in career choice. They should send their ward in teacher education if they convinced that he/she can be a real teacher and can do justice in the field. There should be mindset and valuing the teachers in the society. iv) Internal customer (teachers) In the modern society, the satisfaction of internal customer is as important as that of external customer. In order to get value from outside any system first should be valued from within because poor internal relationship will definitely prevent the institution from working properly and ultimately external customer suffers. Indicators of quality assurance i) Organizational mission The mission of an institution is its basic purpose and reason for existence. The mission of an institution should be articulated in a mission statement, understood by all the teachers. Valued by them and used consistently to guide all plans, goals and action. For example, mission of a ashram school could be empowerment of vulnerable section of society. ii) Continuous improvement Critical thinking and innovations, openness and flexibility. iii) Student orientation Quality implies fulfilling and exceeding the expectations of students in particular and the larger society in general. iv) Leadership commitment Commitment is in terms of emphasis on the value and importance of quality, allocation of resources and support for quality and building commitment among all the staff members towards quality. v) 
Empowerment Empowerment is helping teachers to use their personal, professional or situational powers that they possess.

vi) Collaboration Collaboration teams include members from different departments, subjects levels etc. so as to utilize divers viewpoints, knowledge and skills of such people. vii) Focus on processes Failures in quality are the result of faulty processes rather than faulty human beings. processes are a set of actions carried out to achieve predetermined results. Processes are generally complex and are linked to preceding and following actions. i.e., processes are linked to inputs (preceding actions) and outputs (following action) of an institution. For enhancing the quality of processes, it is necessary to ensure the consistency, validity, reliability, usability and cost-effectiveness of the processes.

According to national curriculum framework 2005, list vision for teacher education.

Teacher education must become more sensitive to the emerging demands from the school system. For this it must prepare the teacher -

1) To care for children, and should love to be with them. 2) To understand children within social, cultural and political contexts. 3) Be receptive and be constantly learning. 4) View learning as a search for meaning out of personal experience, and knowledge generation as a continuously evolving process of reflective learning. 5) View knowledge not as an external reality embedded in textbooks, but as constructed in the shared context of teaching-learning and personal experience. 6) Own responsibility towards society, and work to build a better world. 7) Appreciate the potential of productive work and hands on experience as a pedagogic medium both inside and outside the classroom. 8) Analyze the curricular framework, policy implications and texts.

Conclusion: Quality assurance in teacher education plays the central role in the total quality management mission of the system. Quality assurance can be ensured by its application in all parts of customers. The main focus is on primary customers i.e. learners. Similarly equal attention need to be paid on the total education system and slight deviation in any part of sub-system will disturb the way of quality assurance.

The importance of the Quality Indicators and their proper identification and definition assumes the greatest importance in the context of continuous improvement and quality assurance. The key dimensions that reflect quality can be translated into indicators of quality. These serve two main purposes of the institutions

1. To measure the processes and their outcomes for making necessary adjustments and changes for quality improvement of

the

programme.

2. As an appropriate tool for monitoring the processes for continuous learning and ongoing improvement. The underlying value is to prompt investigations that in turn provide insights into the quality provision of the institution and its programmes leading to continuous improvement. Some of the aspects necessary for effectively putting to use these quality indicators for ongoing improvement of the teacher education programme such as: how they can be used to measure multiple factors, use multiple techniques, involve people from all levels and units, Integrate the information from related indicators to provide complete picture of the institution, , adequate education and training to the members of the institution for identifying and using indicators are some of the issues which will be covered in this brief session. The quality indicators are expected to be tools that prompt additional inquiry and data/information for making necessary adjustments for continuous improvement. The approach is not for definitive judgments on quality and student 
development. The information and data on each indicator would help institution standardize data on relevant areas of improvement, thereby providing a base for future plans through stratification and necessary adjustments in the processes. Comparing and contrasting using the data/information across the institution and with other providers will help in effectively ranking those aspects of teacher education that have a potential for improvement The tools and approaches to translate the key quality aspects of the teacher education programmes into quality indicators would be discussed. As some of the indicators are interpreted as the areas, activities or processes on which evidence need to be collected through interviews, feedback sessions, interactions or validation of documented information, an orientation for the application and use of multiple techniques for using the quality indicators will be built into one of the sessions.

Finally a feasible approach for carrying out a self-assessment of the teacher education programme would be discussed and evolved for adoption and trying out.

Increasing societal concern about the perceived decline of moral and ethical values in contemporary life is promoting renewed interest in moral education or character education (Beck, 1990; Chazan, 1985; Cohen, 1995; Jarrett, 1991; Kelsey, 1993; Lickona, 1991; Nucci, 1989; Ryan \& McLean, 1987; Spiecker \& Straughan, 1988; Wynne \& Ryan, 1993). Some argue that the current educational climate has contributed to a culture in which many do not know what a genuinely moral standard is (Delattre \& Russell, 1993, p. 24); they believe that by removing such words as right and wrong from the school vocabulary, schools have been values-neutral for so long that our ability to engage students in conversations about moral issues had become rusty. We were not even sure what our role was in the moral education of our students (Gecan \& Mulholland-Glaze, 1993, p. 46). Others, exploring the moral and ethical dimensions of schools, claim schooling is a moral endeavor by its very nature (Goodlad, Soder, \& Sirotnik, 1990; Jackson, Boostrom, \& Hansen, 1993; Kirschenbaum, 1994; Sergiovanni, 1992, 1996; Sockett, 1993).

Both groups acknowledge the growing public demand that schools more directly stand for, reflect, and impart valued principles (Cohen, 1995; Lickona, 1991; Wynne \& Ryan, 1993). This recognition, accompanied by support for formal character education or not, has been fueled in part by the greater acceptance of the belief that--regardless of our diversity-at root we share a basic morality that includes such virtues as responsibility, respect, trustworthiness, fairness, caring, and civic virtue (Sergiovanni, 1996, p. 123).

Strike and Ternasky (1993) identify three areas in education to which ethics apply: deliberation and reflection on educational policy, moral education, and professional ethics. The last area, which Strike and Ternasky describe as the most neglected until recently, provides the focus for the following discussion.

In this article, I connect conceptually the professional ethics of teaching and moral education by exploring the function of teacher education to prepare teachers to understand the moral and ethical complexities of their role and thus enable them to reflect ethical actions and decisions in their professional practice. Teachers' practice inevitably has a strong influence on the moral lessons students directly and indirectly acquire in the classroom (Jackson et al., 1993). To be guides for the young in morality and ethics, teachers must understand the complex moral role that they occupy as ethical professionals and appreciate the significance of their own actions and decisions on the students in their care. Moral education is a term applicable to the preparation of future teachers, as much as to children and adolescent students (Bricker, 1993). The recognition that enhanced awareness of teachers of their own ethical practice can be a powerful force on moral education as it evolves in schools.

In the following sections, I review briefly recent theoretical attention to the concept of the teacher as moral agent and exemplar; consider the implications of this concept for teacher education, specifically related to a reconceptualization of foundations courses in educational philosophy and policy; explore the case study method to the teaching of applied ethics; and, using examples from my own practice, provide an overview of potential moral and ethical dilemmas in teaching that some preservice teachers identified in their interpretations of professional and practical experiences.

The Teacher as Moral Agent and Exemplar

Recent educational literature has focused on the teacher's role as fundamentally concerned with the state of moral agency (Fullan, 1993; Grace, 1995; Sergiovanni, 1992, 1996; Sockett, 1990, 1993; Strike \& Ternasky, 1993; Strom, 1989). Some argue that the components of teaching as a knowledge endeavor and as a moral enterprise are essentially inseparable and that recognition of this fusion must be central to the conception of the teacher's role (Ball \& Wilson, 1996). Bull (1993) notes that this renewed conviction that teaching itself has an inherent moral dimension (p. 70) provides a rationale for the inclusion of ethics in preservice teacher education programs. Jackson et al. (1993), in their study of the moral life of classrooms and schools, refer to teachers as moral agents whose influence extends to what they say and do without 
consciously intending to act as moral agents (p. 237). Moral agency does not pertain simply to the deliberate decisions of the self-conscious moral educator but characterizes many of the unintentional forces of teaching.

The need to prepare teachers for working in multicultural contexts in an increasingly globalised world is one that all of us working in education would acknowledge as being of significance. It is something that particularly exercises us on the Development and InterCultural Education (DICE) Project, as we are concerned with both development and intercultural education.

DICE works with student teachers in the four Dublin colleges of education (primary) and has links with Mary Immaculate College in Limerick. The Project delivers courses in development and intercultural education, some of which are elective while other courses are a compulsory component of an Inclusive Education Module in Years 2 and 3 of the BEd in the Trinity-associated colleges (Coláiste Mhuire, Froebel College and The Church of Ireland College of Education). This paper will explore some of the challenges that we have encountered in our work in initial teacher education, focusing on our input with student teachers, and will also draw on some of our experiences of working with college lecturers and practising teachers. The paper will also describe some of the responses that we have developed to these challenges.

\section{Challenges and responses in relation to content and focus}

Within preparing teachers for working in an increasingly globalised world, there are two competing demands on course design and on the focus of delivery. Mainly that there is a danger that the urgency of 'local' needs can push an exploration of the 'global' off the agenda or down the list of priorities. The immediacy of these needs means that it is critical for DICE to have a response to these issues as they arise but also to consider the project's primary task of promoting both development and intercultural education.

One of the on-going challenges therefore, is to ensure that preparation for the 'multicultural context' does not take precedence over a development education perspective. There can be a tension between the dual demands of 'development education' and 'intercultural education', and the perceived urgency of the intercultural agenda can heighten this tension.

In acknowledgement of that tension when DICE commissioned a Literature Review in 2005, it was entitled Global and Justice Perspectives in Education. Early on it emerged that little, if any, literature used the focus or concept of 'global and justice perspectives'. Rather, literature focused more specifically on either development /global, intercultural or citizenship education. We identified ten core themes in the field of development/global; intercultural and citizenship education:

1. Development

2. Sustainable development

3. Interdependence

4. Cultural identity and diversity

5. Human rights and responsibilities

6. Discrimination, racism, prejudice

7. Equality and social justice

8. Peace, conflict and conflict resolution

9. 'State of the world' - geographic, economic, political, social, and environmental knowledge

10. Migration

By working with these ten core themes, practitioners are drawing the strands of development and intercultural education together under a common framework in which the local and the global become intertwined and interconnected.

Another response lies in course design. In recognition of the immediacy of students' concerns with local issues we begin the course with a module exploring just that. Immediate concerns can be used as a 'way in' and also as a means of underlining the importance of this work with students. Courses or sessions then move beyond the local and extend into looking at development education themes such as exploring the concept of 'development'; teaching about distant localities; teaching about 'natural' disasters; human rights education and so on. However, while this approach provides an entry point with students, it is also possible that the interest of many students will not extend past practical and immediate classroom concerns of incorporating global awareness into their teaching. This leads to the all-important question, "what makes a global teacher?". 
The examples that follow are drawn from evaluations on completion of an eight to ten-week module delivered to Year 2 BEd students. Often students drew from their Teaching Practice experiences. Student comments on this first session in their end-of-term evaluations included:

"It is a good idea to have clear information on various cultural differences and I learned a lot about the various cultures in Ireland to aid me when teaching children of various cultures".

"As Ireland is changing so rapidly, it is important that teachers promote equality to the next generation".

"I wouldn't have really thought there was a major need for intercultural education so this lecture opened my eyes to the need for this type of education".

\section{Classroom application: The development of skills}

The following comments highlight a point often made by students on DICE courses. That is that the classroom application of ideas and concepts is of paramount importance to them and is often the deciding factor about whether they have found a session useful or not. For example:

\section{Teaching about 'natural' disasters:}

"....I thought it could be very useful topic to integrate into History, Geography, SPHE, English".

Simulation activity on food/population distribution globally:

"...I will try this activity during my next TP [teaching practice] as I think it conveys the message of unequal distribution of food very clearly".

"The game was great, very hands-on and useful to use in the classroom, really showed powerfully the reality of the division of food".

While this is a very small sample of student evaluations, and certainly more structured investigation would be required over a period of time, it is consistent with feedback that DICE regularly receives from students on completion of courses. It is often apparent that what matters first and foremost to students is the potential applicability of the material to teaching; if they can use it in the classroom then it is valuable. Whether that relates to development or intercultural education appears to be of secondary concern, for example:

"A lot of ideas given for an 'ideal world' but not many for teaching practice which is priority for all at the moment" (Overall course comment).

"(I would like) more designing and planning our own lessons and approaches" (Overall course comment).

A student may respond very positively to particular practical aspects of a course, but a longitudinal study would be required in order to see if this interest was maintained past the particular lesson activity or topic. Furthermore, DICE cannot shape a course purely around the practical application of ideas. Development and intercultural education is concerned with knowledge and attitudes as much as with skills, so it is imperative that we build students' knowledge base in courses. This is despite feedback that shows that theoretical discussions are often less popular with students:

"Found it hard to relate the ideas to the classroom" (Feedback on 'Exploring Culture').

"Interesting, surprising facts; not classroom usable though!" (Feedback on 'Development' session).

"Found that it was too theoretical too soon." (Feedback on 'Exploring Culture').

Attitudinal development: "What makes a 'global teacher'?"

In addition to knowledge and skills, the third aspect which DICE courses aim to develop is attitudinal. This leads back to the earlier question: "What makes a global teacher?" In other words, why is it that some students will go on to incorporate a global and justice dimension as an integral part of their teaching, while another student who may have attended the same courses, will see this aspect as peripheral or irrelevant to their practice? The answer possibly lies in this third area of attitudes. The motivational factor is the key to whether a student will a) build on her /his knowledge base in relation to development and intercultural education and b) acquire the necessary skills to incorporate the perspective into teaching. Some of the students will have learnt interesting activities which they may use as one-off or sporadic exercises. Others will go on to develop an approach to their entire teaching career.

Some of the material in the Literature Review Global and Justice Perspectives in Education (2005) is useful to consider in the context of motivation and attitude. The literature review presented and analysed Irish and international literature on the factors which influence teachers' incorporation of global and justice perspectives in their teaching. As would be expected, whole-school issues such as ethos and leadership figure strongly as facilitating factors, as do aspects such as time, make-up of the student body, and the availability of resources. 
The DICE literature review has found that certain life experiences can influence a teacher's value system and how they incorporate this into their professional environment. This in turn can determine the extent to which their teaching methodologies reflect global and justice perspectives. The researcher cites her own research with practising teachers (Fitzgerald, 2003) to highlight this:

"For those teachers who did not have the same life experiences, they did not exhibit the same awareness of social injustice, or a desire to bring about change in society...The underlying cause of this differentiation between the two groups of teachers was found to lie in a differing value system between the teachers, arising from having different personal experiences in life" (Fitzgerald, 2003, quoted in Literature Review, DICE 2005).

Nevertheless, we cannot assume that the only students who will go on to incorporate a global dimension are those who already have a disposition towards these values as they commence their BEd. We must also hope that our input will have a positive impact on the attitudes of many students, including those not previously disposed. The attitudinal component of DICE courses therefore remains of central importance as well as being one of the most challenging aspects of the work. Part of that challenge is the need to engage students at an emotional as well as at a cognitive level, often within the constraints of relatively short courses.

Is emotional engagement possible over one term, with students meeting in large groups for one hour each week? Interestingly, some of the feedback would suggest that some attitudinal changes were occurring:

"I think that if anybody had any prejudices before going to these lectures, the lectures would probably open their minds more..." (Overall course comment).

"Really opened my eyes as to how unfair the world is..." (Session on Food).

"Seemed very obvious initially but made me think much more deeply over the following weeks of the issues raised" (Introductory session).

These comments suggest that including an experiential and discursive dimension in sessions is a critical response to the challenge of addressing attitudinal issues. Further work would be required to ascertain whether the students who responded in the way described above already have a predisposition towards social justice issues. A minority of student responses would indicate however that engaging with concepts such as social justice is a little more difficult:

"Introduction task (visualisation about moving to a new country) was very drawn out and required much too high a level of concentration than most of us had at that time of the day" (Session on migration).

"I find the content is strongly associated with Geography and therefore should not have so much time allocated to it" (Overall course comment).

\section{Conclusion}

Preparing teachers for working in multicultural contexts in an increasingly globalised world is one of the crucial challenges facing teacher educators today. Attitudinal factors are fundamental to this, and while life experiences may be central to attitudinal change, college courses can support this work in a very real way. Courses on development and intercultural education may challenge some students' attitudes yet may also provide essential support for those students who already have a predisposition towards issues of social justice. For those students, DICE sessions may provide a dedicated space in which to explore matters of importance to them, about equality and justice, whether local or global, and gain tools for incorporating these issues into their future teaching.

This small scale evaluation of student responses to DICE courses reveals the need for a more structured longitudinal study which could explore the issues in greater depth. In 2005-2006 the DICE Project administered a baseline survey to students undertaking modules in development and intercultural education across some of the colleges of education, the results of which are being analysed at present. This will provide some insight into students' knowledge of and attitudes towards social justice perspectives in the second and third years of the BEd, opening the way for follow-up work to take place. It will also inform our thinking about further design and development of modules. It is crucial for teacher educators, especially in the area of social justice, to continue to develop our courses in order to best educate student teachers for working in increasingly diverse settings, in the wider context of a globalised world.

\section{References}

[1] Claxton, G (1999) Wise Up: the Challenge of Lifelong Learning, London, Bloomsbury

[2] DICE (2005) Global and Justice Perspectives in Education: A Literature Review, DICE, Dublin.

[3] Fitzgerald, H (2003) Intercultural Education in the Irish Primary School System - A Teachers' Perspective, Unpublished MA Thesis, University of Limerick. 


\section{ISSN:2321-1091}

[4] Lyle, S \& Salmon, C (2003) 'The Global Dimension in Initial Teacher Education: A Case Study of Twelve Mentors and Twelve ITE Students Following BA (Ed) Degree Programme', Paper presented at UNESCO Conference on Teaching and Learning for Intercultural Understanding, Human Rights and a Culture of Peace,Jyvaskyla, Finland, 15-18th June, 2003.

[5] Inman, S (2005) Making Values Central: The 'Partners in Change' Project, The Development Education Journal, Vol. 11, no.2. 